\title{
Ameliorating Effect of Transcutaneous Electroacupuncture on Impaired Gastric Accommodation in Patients with Postprandial Distress Syndrome-Predominant Functional Dyspepsia: A Pilot Study
}

\author{
Feng Xu, ${ }^{1}$ Yan Tan, ${ }^{2,3}$ Zhihui Huang, ${ }^{3,4}$ Nina Zhang, ${ }^{3}$ Yuemei Xu, ${ }^{1}$ and Jieyun $Y i{ }^{3}$ \\ ${ }^{1}$ Division of Gastroenterology, Yinzhou Hospital Affiliated to Medical School of Ningbo University, Ningbo 315000, China \\ ${ }^{2}$ Division of Gastroenterology, Affiliated Hospital of Hainan Medical College, Haikou 571000, China \\ ${ }^{3}$ Ningbo Pace Translational Medical Research Center, Beilun, Ningbo 315000, China \\ ${ }^{4}$ Department of Gastroenterology, Sir Run Run Shaw Hospital, School of Medicine, Zhejiang University, Hangzhou 310000, China \\ Correspondence should be addressed to Feng Xu; xufengxh19@163.com and Jieyun Yin; jieyunyin07@gmail.com
}

Received 18 July 2014; Accepted 2 September 2014

Academic Editor: Jiande Chen

Copyright (c) 2015 Feng Xu et al. This is an open access article distributed under the Creative Commons Attribution License, which permits unrestricted use, distribution, and reproduction in any medium, provided the original work is properly cited.

\begin{abstract}
Patients with functional dyspepsia (FD) have both reduced gastric accommodation and impaired gastric motility that are difficult to treat. The aim of this study was to investigate the therapeutic potential of transcutaneous electroacupuncture (TEA) for both of these disorders in FD patients. Acute experiments were performed in FD patients to study the effect of TEA and sham-TEA on gastric accommodation assessed by a nutrient drink test and gastric motility assessed by the measurement of the electrogastrogram (EGG). TEA or sham-TEA was performed via cutaneous electrodes at acupoints ST36 and PC6 or sham-points nonacupoints. It was found that (1) gastric accommodation (maximum tolerable volume) was reduced in FD patients compared with the controls $(P<0.03)$. TEA improved gastric accommodation in FD patients $(P<0.02)$. (2) Acute TEA significantly increased the percentage and power of normal gastric slow waves in the fed state assessed in the FD patients by the EGG in comparison with sham-TEA. (3) TEA increased vagal activity assessed by the spectral analysis of the heart rate variability in the fed state in FD patients. It was concluded that needleless method of transcutaneous electroacupuncture may have a therapeutic potential for treating both impaired gastric accommodation and impaired gastric motility in patients with FD.
\end{abstract}

\section{Introduction}

The prevalence of functional dyspepsia (FD) is high but the treatment options have been limited [1]. Patients with FD complain about symptoms of epigastric pain, abdominal fullness, early satiety, and abdominal discomfort. Pathophysiologies of FD include visceral hypersensitivity, reduced gastric accommodation, and impaired gastric motility, such as gastric dysrhythmia, antral hypomotility, and delayed gastric emptying [2].

Gastric accommodation is mediated by the vagal nerve. Upon food ingestion, the vagal nerve is activated and nitric oxide is released, resulting in a relaxation of the stomach. This relaxation reflex accommodates ingested food without causing an increase in gastric pressure [3]. Impaired gastric accommodation leads to early satiety and postprandial fullness, possibly attributed to weakening of the vagal nerve.

After the patients with GI disorder eat food, a series of indigestion symptoms of early satiety and abdominal distension will appear due to insufficient relaxation of proximal gastric and intragastric pressure increasing. About $40 \%$ to $70 \%$ of FD patients have proximal GI disorder [4]. Accordingly, treatment for impaired gastric accommodation is of great clinical significance $[5,6]$.

Common treatment options for FD include dietary measures, pharmacologic treatments, such as acid-suppression drugs, prokinetic agents, fundus relaxing drugs, and antinociceptive agents, and psychological interventions [7-16]. In 
general, targeted therapies directed at the underlying pathophysiology are desirable. However, efficacy of the therapy is usually very limited due to multiple symptoms and pathophysiologies in individual patients. For example, a patient may have impaired accommodation and delayed gastric empting at the same time; in this case, prokinetic agents can be used to treat delayed gastric emptying but would worsen the symptoms related to gastric accommodation because available prokinetics often impair gastric accommodation. For the same reason, fundus relaxing drugs may be used for treating impaired accommodation; however, these drugs may delay gastric emptying because they relax muscles. The treatment approach to the patients with hypersensitivity to gastric distension has not been established. Antidepressants are commonly used in functional gastrointestinal disorders and were thought to exert a visceral analgesic rather than an antidepressant effect. However, studies of the effects of antidepressants on visceral sensitivity are rare, and the existing data on visceral sensitivity are controversial [14, 15].

Acupuncture has been used to treat gastrointestinal symptoms in China for thousands of years. The most commonly used acupuncture points (acupoints) for the treatment of gastrointestinal symptoms are Neiguan (PC6) and Zusanli (ST36). In clinical research, manual acupuncture is commonly replaced with electroacupuncture that is more reproducible. In a comparative study, electroacupuncture was found to be as effective as manual acupuncture in treating pain [17]. Electroacupuncture at ST36 and PC6 has been documented to increase the regularity of gastric slow waves and accelerate gastric emptying of liquids in animals [18]. In recent studies, electroacupuncture was reported to accelerate gastric emptying of solids and improve dyspeptic symptoms and gastric dysrhythmia in patients with FD and patients with diabetes $[19,20]$ and similar beneficial effects can be observed in patients with FD when electroacupuncture is applied without needles or a method called transcutaneous electroacupuncture (TEA) [21]. TEA is a completely noninvasive method which is readily accepted by patients. However, it is unknown whether TEA is able to treat both reduced gastric accommodation and impaired gastric motility in patients with FD.

The aims of this study were to investigate the therapeutic potential of TEA for patients with FD by assessing its acute effects on gastric accommodation assessed by a noninvasive nutrient drink test and gastric motility assessed by noninvasive electrogastrography as well as dyspeptic symptoms and to explore vagal mechanisms involved with TEA.

\section{Materials and Methods}

2.1. Subjects. Eight FD patients with postprandial distress syndrome and 8 healthy volunteers aged 21 to 65 years old were recruited in this study. Patients included fulfilled Rome III criteria for FD postprandial distress syndrome [1]. Patients who were unable to give informed consent; were taking prokinetic, anticholinergic, or dopaminergic agents during the experimental period; had a history of gastrointestinal surgery; were pregnant or preparing to conceive a child; had diabetes; and were allergic to skin preparation and familiar with acupoints and their functions were excluded from the study. Inclusion criteria of healthy volunteers include no history of supreme gastrointestinal diseases, including peptic ulcer disease, gastroesophageal reflux disease, and hepatobiliary and pancreatic diseases, a history of abdominal surgery, no history of alcohol abuse, no serious systemic illness and possible malignancy, and usually no dyspeptic symptoms, including upper abdominal pain, upper abdominal discomfort, postprandial fullness, upper abdominal swelling, early satiety, nausea, vomiting, excessive belching, and heartburn. All general information including height, weight, address, and relating medical history is recorded and all the subjects had signed the informed consent prior to the study. The experimental protocol was approved by the ethical committee of Yinzhou People's Hospital and all the subjects signed the consent form before participation.

2.2. Experimental Protocol. All subjects were studied in the morning after a 12-hour fast. Each subject was studied for two sessions in a randomized order: TEA and sham-TEA sessions. The experiment protocol was as follows: 30-minute baseline recording, 30-minute TEA/sham-TEA treatment in the fasting state, and then a satiety drinking test conducted with a liquid meal of Ensure $(0.95 \mathrm{kcal} / \mathrm{mL})$ with TEA/ShamTEA. After the completion of satiety drinking test, there was a 30-minute recovery period with TEA/sham-TEA. Electrogastrogram (EGG) and electrocardiogram (ECG) were recorded during the entire experimental period except during the satiety drinking test.

2.3. Transcutaneous Electroacupuncture. Acupoints ST36 (Zusanli) and PC6 (Neiguan) were used in the TEA session. ST36 is located at the place of 4-finger-breadth measuring down from the outer eye of the knee between the fibula and the tibia, 1-finger-breadth measurement beside the tibia; PC6 is located at the place of one-sixth of remote end and five-sixths of proximal end of the connection stripe between the transverse wrist crease and cubical crease. The stimulation was delivered by two portable neuromodulation devices at ST36 and PC6, respectively (SNM-FDC01, Ningbo Maida Medical Device Inc.). The stimulation parameters were chosen as 2s-on, 3s-off, $25 \mathrm{~Hz}, 0.6 \mathrm{~ms}$, and amplitude of $2 \mathrm{~mA}$ to $10 \mathrm{~mA}$ depending on tolerance of the subject, which was shown to improve gastrointestinal symptoms in patients with diabetic gastroparesis [22]. In the sham-TEA group, the sham-acupoint for PC6 was located at about 15-20 cm away from PC6 (up to the elbow and outside coastal margin of the forearm not on any meridian) and the sham-point for ST36 was located at $10-15 \mathrm{~cm}$ down from and to the lateral side of ST36 not on any meridian. The stimulation parameters used for sham-TEA were the same as in the TEA.

2.4. Satiety Drinking Test. The gold-standard method of assessing gastric accommodation is the barostat method. However, this method is not well tolerated by patients due to intubation of a plastic bag into the stomach. Recently, the satiety drinking test has been used as a surrogate for the measurement of gastric accommodation [23]. A higher volume taken by the subject is indicative of a higher gastric 
accommodation. In this method, after an overnight fast, the subject was instructed to take Ensure $(0.95 \mathrm{kcal} / \mathrm{mL})$ at a rate of $120 \mathrm{~mL}$ every 4 minutes (average $30 \mathrm{~mL} / \mathrm{min}$ ) until the subject reported to reach satiety (complete fullness). During the test, each subject was asked to score satiety at a 5minute interval using following scores: 0 : no symptoms; 1 : initial satiety (threshold); 2: mild; 3: moderate; 4: severe; 5: maximum or intolerable satiety. When reaching score 5 , the subject was asked to stop drinking and the total volume drunk was recorded, which reflected the maximum tolerable volume (MTV).

2.5. Assessment of Autonomic Function. The electrocardiogram (ECG) was recorded using a one-channel amplifier with a cut-off frequency of $100 \mathrm{~Hz}$ (Ningbo Maida Medical Device Inc., Ningbo, China) from two active ECG electrodes and one ground electrode. The two leads were attached to the right edge of the sternum and apex of the subjects and the ground to the right side of the abdomen. The heart rate variability (HRV) signal was derived from the ECG recording using a special program developed [24] by identifying $\mathrm{R}$ peaks, calculating and interpolating the R-R intervals so that the time interval between consecutive samples was equal and finally downsampling the interpolated data to a frequency of $1 \mathrm{~Hz}$.

Overall power spectral analysis was applied to the HRV signal and the power in each frequency subband was calculated. The power in the low frequency band (0.04$0.15 \mathrm{~Hz}$ ), LF, represents mainly sympathetic activity and part of parasympathetic activity. The power in the high frequency band $(0.15-0.50 \mathrm{~Hz}), \mathrm{HF}$, stands purely for parasympathetic or vagal activity. For LF and HF, standard calculations were done, respectively, by $\mathrm{LF} /(\mathrm{HF}+\mathrm{LF})$ and $\mathrm{HF} /(\mathrm{HF}+\mathrm{LF})$ [25].

2.6. Recording and Analysis of Electrogastrogram (EGG). The gastric myoelectrical activity was recorded using a 4-channel electrogastrogram (EGG) device (MEGG-04A, Ningbo Maida Medical Device Inc., Ningbo, Zhejiang, China) via 6 cutaneous electrodes described as follows. First, the abdomen where electrodes were to be placed was cleaned with a special gel (Nuprep, Weaver and Company, Aurora, USA); then conductive gel (Ten20, Weaver and Company, Aurora, USA) was applied to the cleaned skin area to reduce skin-electrode impedance. After this, six cutaneous electrodes were placed on the abdominal skin surface based on a previously established method [2]. The subject was in a supine position for the EGG recordings and talking, reading, or sleeping was not allowed.

Established EGG parameters were derived from the EGG signals using a spectral analysis software package (Ningbo Maida Medical Device Inc., Ningbo, China) after a careful deletion of motion artifacts [26, 27]: (1) dominant frequent and power, representing the frequency and amplitude of gastric slow waves; (2) percentage of normal 2-4 cycles/min slow waves, representing the regularity of gastric slow waves; (3) postpreprandial ratio of EGG dominant power, standing for postprandial increase in gastric motility.
TABLE 1: Effects of acute TEA treatment on EGG in patients with functional dyspepsia in the study.

\begin{tabular}{lcc}
\hline & \multicolumn{2}{c}{ Session } \\
& TEA & Sham-TEA \\
\hline Dominant frequency $(\mathrm{cpm})$ & $3.02 \pm 0.03$ & $3.04 \pm 0.06$ \\
$\quad$ Fasting & $2.84 \pm 0.07$ & $3.25 \pm 0.10$ \\
$\quad$ Postprandial & & \\
Dominant power $(\mathrm{dB})$ & $33.98 \pm 1.58$ & $34.46 \pm 1.75$ \\
$\quad$ Fasting & $42.35 \pm 1.35$ & $40.24 \pm 1.47^{*}$ \\
$\quad$ Postprandial & & \\
Percentage of normal slow waves $(\%)$ & & \\
$\quad$ Fasting & $82.6 \pm 3.1$ & $83.7 \pm 2.7$ \\
$\quad$ Postprandial & $85.42 \pm 4.27$ & $74.97 \pm 6.60^{*}$ \\
Postpreprandial power ratio & $1.03 \pm 0.03$ & $0.92 \pm 0.04$ \\
${ }^{*} P<0.05$. & &
\end{tabular}

2.7. Assessment of Dyspeptic Symptoms. Gastric cardinal symptom index was used to assess dyspeptic symptoms at baseline and after the acute TEA or sham-TEA [28]. These included upper abdominal pain, upper abdominal discomfort, postprandial fullness, upper abdominal swelling, early satiety, nausea, vomiting, excessive belching, and heartburn. Each symptom was graded based on severity: grade 0: no symptoms; grade 1: mild; grade 2: moderate; grade 3: severe.

2.8. Statistical Analysis. Results are expressed as mean \pm standard deviation. Paired Student's $t$-test was used to study the difference between TEA and sham-TEA and between baseline and after the acute treatment using SPSS 16.0 statistical software. $P<0.05$ was considered statistically significant.

\section{Results}

3.1. Effects of TEA on Gastric Accommodation. FD patients showed a reduced gastric accommodation that was improved with acute TEA. The MTV was $725 \pm 46 \mathrm{~mL}$ in the normal control group and $548 \pm 38 \mathrm{~mL}$ in the FD patients $(P=0.022$; see Figure 1(a)). Acute TEA increased the MTV in the FD patients to $663 \pm 29 \mathrm{~mL}(P=0.007$, versus baseline), whereas the sham-TEA did not increase the MTV in patients with FD $(549 \pm 36 \mathrm{~mL}$ after sham-TEA $(P=0.121$ versus $700 \mathrm{~mL}))$. There was a difference $(P=0.017)$ in MTV in the FD patients after TEA and sham-TEA (Figure 1(b)).

3.2. Effects of TEA on Gastric Slow Waves. The EGG recording was found to be normal in 2 patients but abnormal in 6 patients with FD (percentage of normal slow waves below $65 \%$ in either fasting or fed state or this was a postprandial decrease in dominant power). The major EGG parameters in the TEA and sham-TEA sessions are shown in Table 1. TEA improved the percentage of normal 2-4 cycles $/ \mathrm{min}$ slow waves in the fed state (Figure 2) and also increased the dominant EGG power in the fed state (Figure 3). 


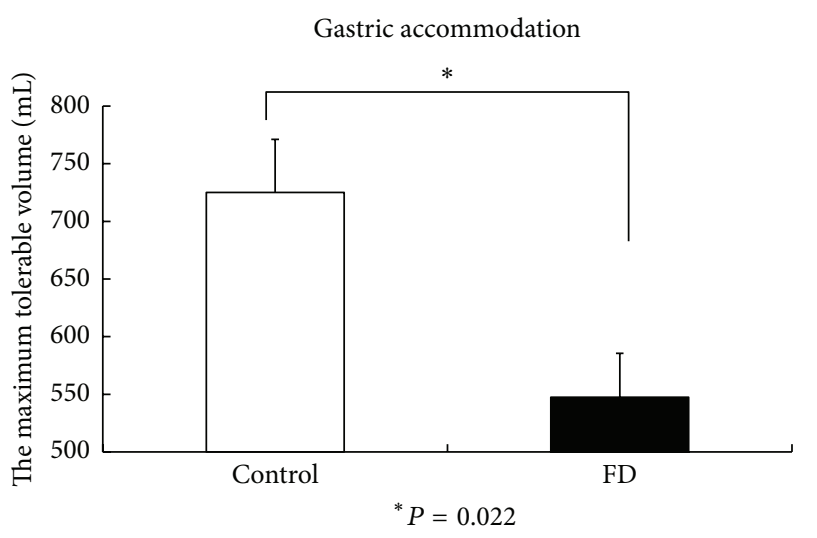

(a)

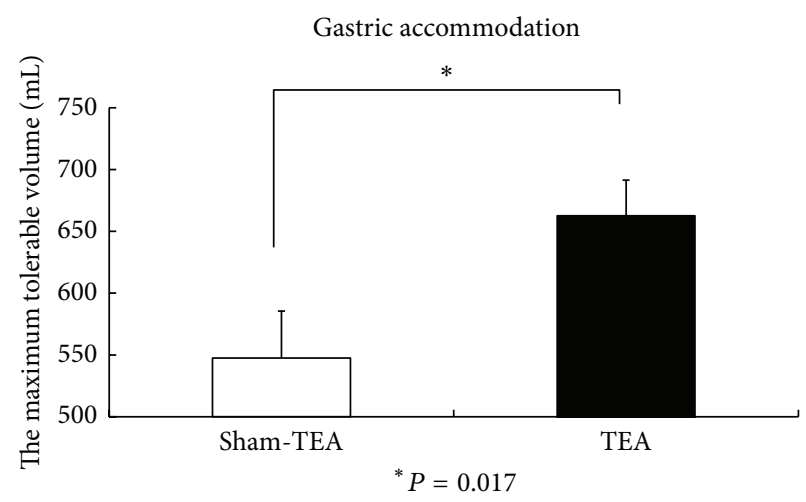

(b)

FIgURE 1: (a) The maximum tolerable volume (gastric accommodation) in normal controls and patients with FD. (b) The maximum tolerable volume after TEA and sham-TEA.

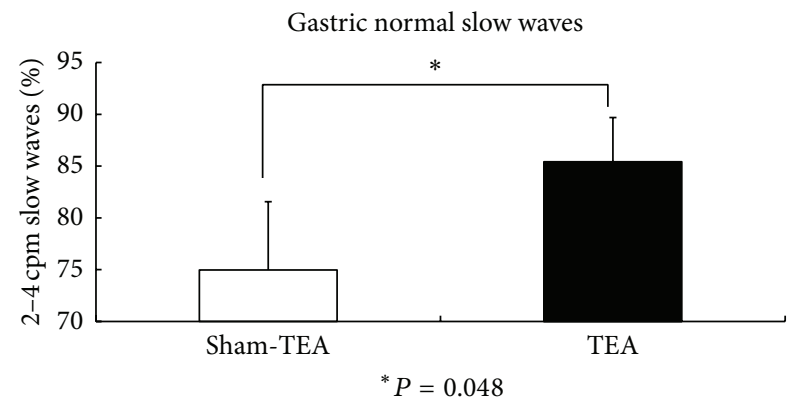

FIGURE 2: TEA improved the percentage of normal 2-4 cycles/min slow waves in the fed state.

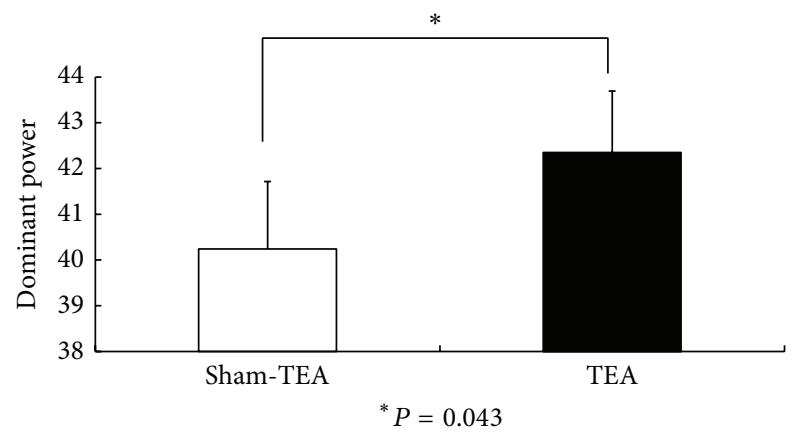

FIGURE 3: The comparison of EGG dominant power in the fed state after sham-TEA and TEA.

The postpreprandial EGG power ratio was significantly higher in the TEA sessions than in the sham-TEA session (Figure 4).

3.3. TEA Enhanced Vagal Activity. The acute TEA significantly increased the vagal activity in the $30 \mathrm{~min}$ postprandial period in patients with $\mathrm{FD}$ assessed by the spectral analysis of HRV. The HF/(LF + HF) was $0.17 \pm 0.01$ in the TEA session

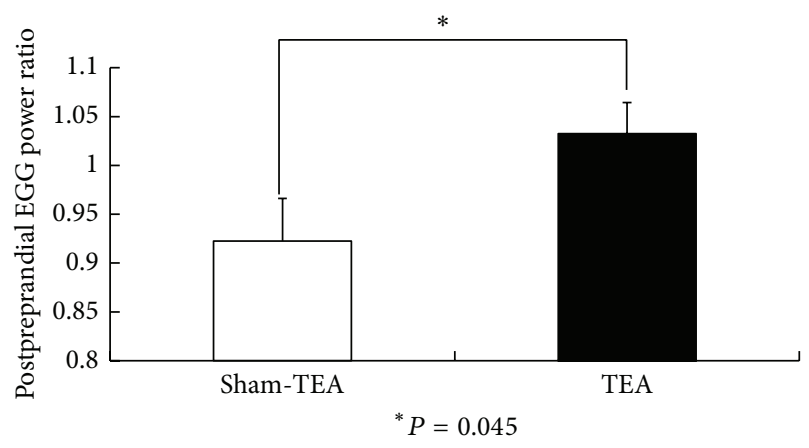

FIGURE 4: The comparison of postpreprandial EGG power ratio between sham-TEA and TEA.

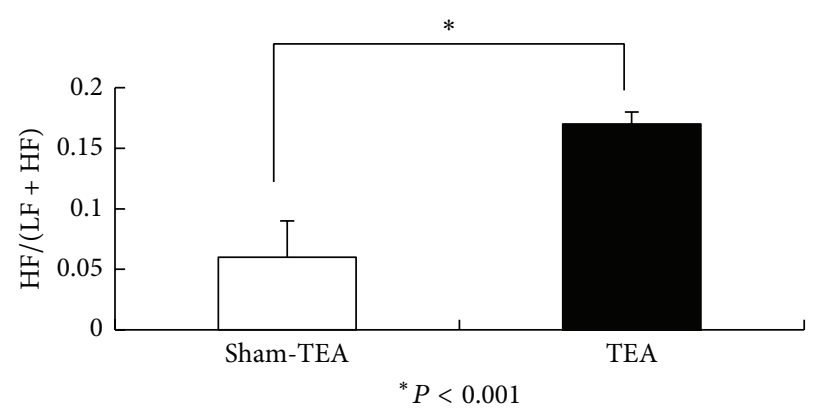

FIgURE 5: The vagal activity HF/(LF + HF) assessed by the spectral analysis of HRV in patients with FD treated with sham-TEA and TEA.

and $0.06 \pm 0.03$ in the sham-TEA session $(P<0.001)$ (see Figure 5).

3.4. Effects of Acute TEA on Dyspeptic Symptoms. Acute TEA improved the dyspeptic symptoms in the FD patients. The mean total symptom score was $23.5 \pm 2.9$ at baseline and decreased significantly to $11.9 \pm 1.4(P=0.007$ versus baseline) after TEA but was $21.9 \pm 2.9$ after sham-TEA 


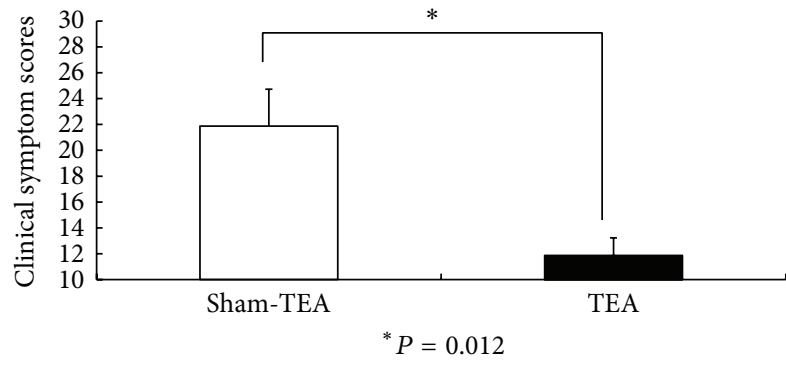

FIGURE 6: The clinical symptom scores in FD patients after TEA and sham-TEA treatment.

( $P=0.102$ versus baseline). There was a significant difference in the clinical symptom scores between the FD patients after true treatment and those after sham treatment (Figure 6).

\section{Discussion}

In this study, we found that acute TEA at the acupoints of ST36 and PC6 improved gastric accommodation and enhanced postprandial gastric slow waves in patients with FD (increased the amplitude and regularity of slow waves). A concurrent increase in vagal activity was also noted with the acute TEA, suggesting a vagal mechanism. Acupuncture or electroacupuncture has been used to treat the symptoms of upper abdomen, such as nausea and vomiting. $\mathrm{Hu}$ et al. [29] reported that electroacustimulation at point PC6 reduced significantly the severity of the symptoms of motion sickness. The number of emetic episodes induced by morphine [30] or cyclophosphamide [31] was significantly reduced by electroacupuncture at the PC6 point in ferrets. Electroacupuncture at both the PC6 and the ST36 points reduced the incidence of vomiting induced by vasopressin in dogs [32]. A few papers reported the effect of acupuncture or electroacupuncture on dyspeptic symptoms in patients with FD. In one study with FD patients, acupuncture was demonstrated to be effective in reducing dyspeptic symptoms [19].

While electroacupuncture has been proven effective in treating certain functional gastrointestinal diseases, the insertion of acupuncture needles is required and the treatment has to be done at a doctor's office. The method proposed in this study, TEA, did not require the insertion of any needles and the procedure could be done by the patient at his/her home. This was more attractive than electroacupuncture and was well accepted by the patients as the compliance of the therapy was $100 \%$; none of the patients quitted the study. It is similar to transcutaneous electrical nerve stimulation except that the stimulation electrodes in this study were placed on the acupuncture points related to the targeting disorder. Liu et al. [33] found that a two-week treatment of TEA at ST36 and PC6 significantly improved dyspeptic symptoms and increased vagal activity in patients with FD. These findings were in agreement with the present study. However, the effect of TEA on gastric accommodation was not previously investigated.

Impaired gastric accommodation in FD is difficult to treat because it requires the use of muscle relaxant. The use of muscle relaxant, however, worsens impaired gastric motility that is common in FD. In this study, acute TEA significantly and substantially improved gastric accommodation while concurrently improving gastric motility assessed by electrogastrography. This is an attractive strength of the proposed method of TEA. As stated earlier, impaired gastric accommodation is associated with symptoms of early satiety and postprandial fullness and bloating. The TEAinduced increase in gastric accommodation could lead to improvement in these symptoms. Although exact mechanisms involved in the increase of gastric accommodation were unknown, the concurrent increase in vagal activity noted in this study was believed to play a major role.

Electrogastrography has previously been shown to be an accurate and reliable method for studying gastric myoelectrical activity. Several studies have reported EGG abnormalities in FD patients [34, 35]. Meanwhile, it is known that electroacupuncture may affect gastric myoelectrical activity. A number of studies have investigated the effect of electroacupuncture on the gastric slow waves. Ouyang et al. [18] showed that electroacupuncture at ST36 and PC6 increased the regularity of gastric slow waves in both the proximal and distal stomach. Chang et al. [20] found that electrical stimulation at ST36 increased the percentage of normal EGG frequency and decreased the percentage of tachygastrial frequency in diabetic patients. Electroacupuncture at ST36 and PC6 increased the percentage of regular slow waves, resulting in the normalization of dysrhythmia in healthy human [36]. However, Liu et al. [33] study showed that TEA at ST36 and PC6 points did not change the EGG parameters in the patients with FD, suggesting that TEA may not treat disorders induced by gastric myoelectrical disturbances. In this study, however, we found that acute TEA at the acupoints of ST36 and PC6 improved gastric slow waves in the postprandial state. It should be noted that in this study the EGG in the postprandial state was recorded after the maximum ingestion of a nutrient liquid meal. This was apparently different from the postprandial recording after a regular meal.

Altered HF and LF/HF in the spectral analysis of HRV in patients with FD have been previously reported [37, 38]. It has been proposed that the autonomic dysfunctions could play a role in the development of disturbed gastric motility and perception. Spectral analysis of the HRV is a noninvasive and simple method for the quantitative evaluation of autonomic activity $[39,40]$. We used this method to evaluate the effect of acute TEA on HRV in patients with FD and found a significant increase in $\mathrm{HF}$ after the TEA treatment. This result is in good agreement with others reported previously [18, 33, 41]. Although we did not have proof that this was responsible for the improvement in dyspeptic symptoms, it was consistent with the hypothesis that the visceral effects of TEA are at least partially mediated by the autonomic nerve pathway.

In summary, acute TEA at ST36 and PC6 significantly improves gastric accommodation and postprandial slow waves as well as dyspeptic symptoms, possibly mediated via the vagal mechanisms. Chronic clinical studies are warranted to establish clinical role of this noninvasive method of TEA for treating FD. 


\section{Ethical Approval}

This work was performed to the principles expressed in the Declaration of Helsinki. This study was approved by the ethical committee in the Yinzhou Affiliated Hospital. An informed consent was obtained from all patients and controls.

\section{Conflict of Interests}

The authors declared no potential conflict of interests with respect to the research, authorship, and/or publication of this paper.

\section{Authors' Contribution}

The work presented here was carried out through collaboration between all authors. Jieyun Yin defined the research theme. Jieyun Yin and Yan Tan designed the methods and experiments. Feng Xu, Yan Tan, Zhihui Huang, Nina Zhang, and Yuemei Xu carried out the clinical experiments and Yan Tan analyzed the data. Yan Tan and Jieyun Yin interpreted the results and wrote the paper. All authors have contributed to and approved the paper. Feng Xu and Yan Tan contributed equally.

\section{Acknowledgments}

This study was partially supported by grants from Beilun District Government and Ningbo Municipal Government.

\section{References}

[1] J. Tack, N. J. Talley, M. Camilleri et al., "Functional gastroduodenal disorders," Gastroenterology, vol. 130, no. 5, pp. 1466-1479, 2006.

[2] X. Lin, D. Levanon, and J. D. Z. Chen, "Impaired postprandial gastric slow waves in patients with functional dyspepsia," Digestive Diseases and Sciences, vol. 43, no. 8, pp. 1678-1684, 1998.

[3] S. Kindt and J. Tack, "Impaired gastric accommodation and its role in dyspepsia," Gut, vol. 55, no. 12, pp. 1685-1691, 2006.

[4] M. W. Mundt and M. Samsom, "Fundal dysaccommodation in functional dyspepsia: head-to-head comparison between the barostat and three-dimensional ultrasonographic technique," Gut, vol. 55, no. 12, pp. 1725-1730, 2006.

[5] O. H. Gilja, T. Hausken, I. Wilhelmsen, and A. Berstad, "Impaired accommodation of proximal stomach to a meal in functional dyspepsia," Digestive Diseases and Sciences, vol. 41, no. 4, pp. 689-696, 1996.

[6] J. Tack, H. Piessevaux, B. Coulie, P. Caenepeel, and J. Janssens, "Role of impaired gastric accommodation to a meal in functional dyspepsia," Gastroenterology, vol. 115, no. 6, pp. 13461352, 1998.

[7] C. A. Maggi, “Therapeutic potential of capsaicin-like molecules: studies in animals and humans," Life Sciences, vol. 51, no. 23, pp. 1777-1781, 1992.

[8] M. Bortolotti, G. Coccia, G. Grossi, and M. Miglioli, "The treatment of functional dyspepsia with red pepper," Alimentary
Pharmacology and Therapeutics, vol. 16, no. 6, pp. 1075-1082, 2002.

[9] N. J. Talley, V. Meineche-Schmidt, P. Paré et al., "Efficacy of omeprazole in functional dyspepsia: double-blind, randomized, placebo-controlled trials (the Bond and Opera studies)," Alimentary Pharmacology and Therapeutics, vol. 12, no. 11, pp. 1055-1065, 1998.

[10] S. Soo, P. Moayyedi, J. Deeks, B. Delaney, M. Innes, and D. Forman, "Pharmacological interventions for non-ulcer dyspepsia," Cochrane Database of Systematic Reviews, no. 2, Article ID CD001960, 2000.

[11] M. D. Gershon and G. M. Jonakait, "Uptake and release of 5hydroxytryptamine by enteric 5-hydroxytryptaminergic neurones: effects of fluoxetine (Lilly 110140) and chlorimipramine," British Journal of Pharmacology, vol. 66, no. 1, pp. 7-9, 1979.

[12] J. Tack, D. Broekaert, B. Coulie, B. Fischler, and J. Janssens, "Influence of the selective serotonin re-uptake inhibitor, paroxetine, on gastric sensorimotor function in humans," Alimentary Pharmacology and Therapeutics, vol. 17, no. 4, pp. 603-608, 2003.

[13] A. B. Gorelick, S. S. Koshy, F. G. Hooper, T. C. Bennett, W. D. Chey, and W. L. Hasler, "Differential effects of amitriptyline on perception of somatic and visceral stimulation in healthy humans," The American Journal of Physiology-Gastrointestinal and Liver Physiology, vol. 275, no. 3, pp. G460-G466, 1998.

[14] P. L. Peghini, P. O. Katz, and D. O. Castell, "Imipramine decreases oesophageal pain perception in human male volunteers," Gut, vol. 42, no. 6, pp. 807-813, 1998.

[15] E. J. Bennett, C. Piesse, K. Palmer, C.-A. Badcock, C. C. Tennant, and J. E. Kellow, "Functional gastrointestinal disorders: psychological, social, and somatic features," Gut, vol. 42, no. 3, pp. 414420, 1998.

[16] S. Soo, P. Moayyedi, J. Deeks, B. Delaney, M. Lewis, and D. Forman, "Psychological interventions for non-ulcer dyspepsia," Cochrane Database of Systematic Reviews, no. 4, Article ID CD002301, 2011.

[17] R. G. Ghaly, K. T. J. Fitzpatrick, and J. W. Dundee, "Antiemetic studies with traditional Chinese acupuncture. A comparison of manual needling with electrical stimulation and commonly used antiemetics," Anaesthesia, vol. 42, no. 10, pp. 1108-1110, 1987.

[18] H. Ouyang, J. Yin, Z. Wang, P. J. Pasricha, and J. D. Z. Chen, "Electroacupuncture accelerates gastric emptying in association with changes in vagal activity," The American Journal of Physiology-Gastrointestinal and Liver Physiology, vol. 282, no. 2, pp. G390-G396, 2002.

[19] S. Xu, X. Hou, H. Zha, Z. Gao, Y. Zhang, and J. D. Z. Chen, "Electroacupuncture accelerates solid gastric emptying and improves dyspeptic symptoms in patients with functional dyspepsia," Digestive Diseases and Sciences, vol. 51, no. 12, pp. 2154-2159, 2006.

[20] C. S. Chang, C. W. Ko, C. Y. Wu, and G. H. Chen, "Effect of electrical stimulation on acupuncture points in diabetic patients with gastric dysrhythmia: a pilot study," Digestion, vol. 64, no. 3, pp. 184-190, 2001.

[21] A. C.-P. Kwan, T. N. Bao, S. Chakkaphak et al., "Validation of Rome II criteria for functional gastrointestinal disorders by factor analysis of symptoms in Asian patient sample," Journal of Gastroenterology and Hepatology (Australia), vol. 18, no. 7, pp. 796-802, 2003.

[22] I. Sarosiek, R. W. McCallum, Y. Sun et al., "Self-administered needleless acupuncture therapy to control dyspepsia and GERD 
symptoms in patients diagnosed with diabetic gastroparesis," Gastroenterology, vol. 144, no. 5, supplement 1, p. S-135, 2013.

[23] J. Tack, P. Caenepeel, H. Piessevaux, R. Cuomo, and J. Janssens, "Assessment of meal induced gastric accommodation by a satiety drinking test in health and in severe functional dyspepsia," Gut, vol. 52, no. 9, pp. 1271-1277, 2003.

[24] Z. S. Wang and J. D. Z. Chen, "Robust ECG R-R wave detection using evolutionary-programming-based fuzzy inference system (EPFIS), and application to assessing brain-gut interaction," IEE Proceedings: Science, Measurement and Technology, vol. 147, no. 6, pp. 351-356, 2000.

[25] C.-L. Lu, X. Zou, W. C. Orr, and J. D. Z. Chen, "Postprandial changes of sympathovagal balance measured by heart rate variability," Digestive Diseases and Sciences, vol. 44, no. 4, pp. 857-861, 1999.

[26] J. D. Z. Chen, R. D. Richards, and R. W. McCallum, "Identification of gastric contractions from the cutaneous electrogastrogram," The American Journal of Gastroenterology, vol. 89, no. 1, pp. 79-85, 1994.

[27] J. D. Z. Chen, W. R. Stewart Jr., and R. W. McCallum, "Spectral analysis of episodic rhythmic variations in the cutaneous electrogastrogram," IEEE Transactions on Biomedical Engineering, vol. 40, no. 2, pp. 128-135, 1993.

[28] J. Tack, A. Masclee, and R. Heading, "A dose-ranging, placebocontrolled, pilot trial of Acotiamide in patients with functional dyspepsia," Neurogastroenterology and Motility, vol. 21, no. 3, pp. 272-280, 2009.

[29] S. Hu, R. M. Stern, and K. L. Koch, "Electrical acustimulation relieves vection-induced motion sickness," Gastroenterology, vol. 102, no. 6, pp. 1854-1858, 1992.

[30] L. Lao, R. H. Wong, B. Berman, and R. L. Wynn, "Electroacupuncture reduces morphine-induced emesis in ferrets: a pilot study," Journal of Alternative and Complementary Medicine, vol. 1, no. 3, pp. 257-261, 1995.

[31] L. Lao, G. Zhang, R. H. Wong, A. K. Carter, R. L. Wynn, and B. M. Berman, "The effect of electroacupuncture as an adjunct on cyclophosphamide-induced emesis in ferrets," Pharmacology Biochemistry and Behavior, vol. 74, no. 3, pp. 691-699, 2003.

[32] J. D. Z. Chen, L. Qian, H. Ouyang, and J. Yin, "Gastric electrical stimulation with short pulses reduces vomiting but not dysrhythmias in dogs," Gastroenterology, vol. 124, no. 2, pp. 401-409, 2003.

[33] S. Liu, S. Peng, X. Hou, M. Ke, and J. D. Z. Chen, "Transcutaneous electroacupuncture improves dyspeptic symptoms and increases high frequency heart rate variability in patients with functional dyspepsia," Neurogastroenterology and Motility, vol. 20, no. 11, pp. 1204-1211, 2008.

[34] A. Leahy, K. Besherdas, C. Dayman, I. Mason, and O. Epstein, "Abnormalities of the electrogastrogram in functional gastrointestinal disorders," The American Journal of Gastroenterology, vol. 94, no. 4, pp. 1023-1028, 1999.

[35] B. Pfaffenbach, R. J. Adamek, C. Bartholomäus, and M. Wegener, "Gastric dysrhythmias and delayed gastric emptying in patients with functional dyspepsia," Digestive Diseases and Sciences, vol. 42, no. 10, pp. 2094-2099, 1997.

[36] X. Lin, J. Liang, J. Ren, F. Mu, M. Zhang, and J. D. Z. Chen, "Electrical stimulation of acupuncture points enhances gastric myoelectrical activity in humans," The American Journal of Gastroenterology, vol. 92, no. 9, pp. 1527-1530, 1997.

[37] S. L. Silva Lorena, M. J. De Oliveira Figueiredo, J. R. Souza Almeida, and M. A. Mesquita, "Autonomic function in patients with functional dyspepsia assessed by 24-hour heart rate variability," Digestive Diseases and Sciences, vol. 47, no. 1, pp. 27-31, 2002.

[38] T. Hausken, S. Svebak, I. Wilhelmsen et al., "Low vagal tone and antral dysmotility in patients with functional dyspepsia," Psychosomatic Medicine, vol. 55, no. 1, pp. 12-22, 1993.

[39] G. G. Berntson, J. Thomas Bigger Jr., D. L. Eckberg et al., "Heart rate variability: origins methods, and interpretive caveats," Psychophysiology, vol. 34, no. 6, pp. 623-648, 1997.

[40] J. Vila, F. Palacios, J. Presedo, M. Fernández-Delgado, P. Felix, and S. Barro, "Time-frequency analysis of heart-rate variability," IEEE Engineering in Medicine and Biology Magazine, vol. 16, no. 5, pp. 119-126, 1997.

[41] M. Tatewaki, M. Harris, K. Uemura et al., "Dual effects of acupuncture on gastric motility in conscious rats," The American Journal of Physiology, vol. 285, no. 4, pp. R862-R872, 2003. 


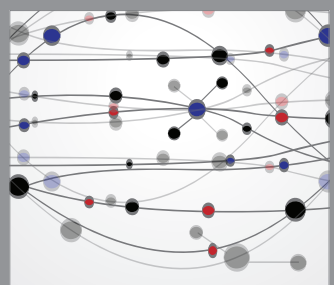

The Scientific World Journal
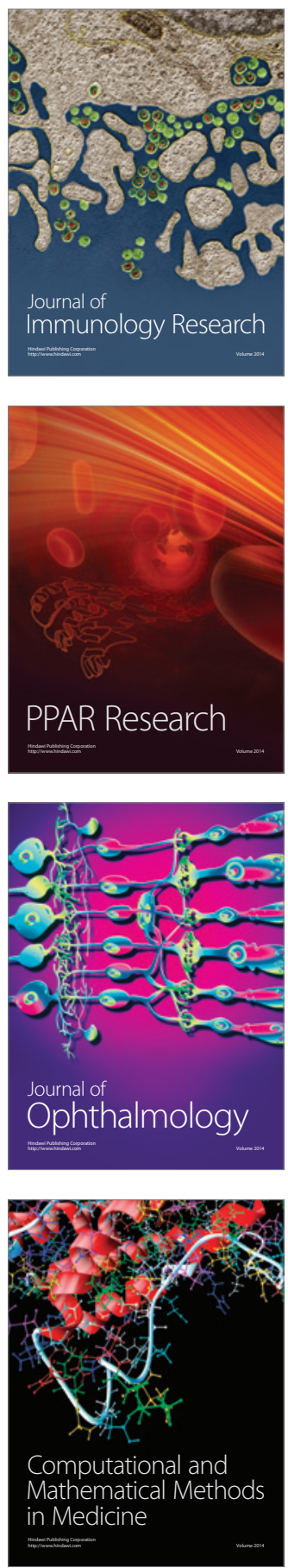

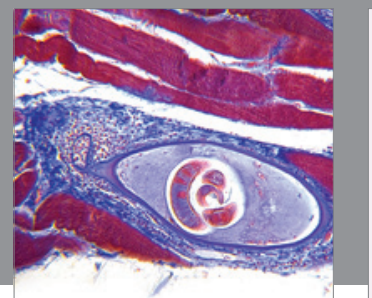

Gastroenterology

Research and Practice
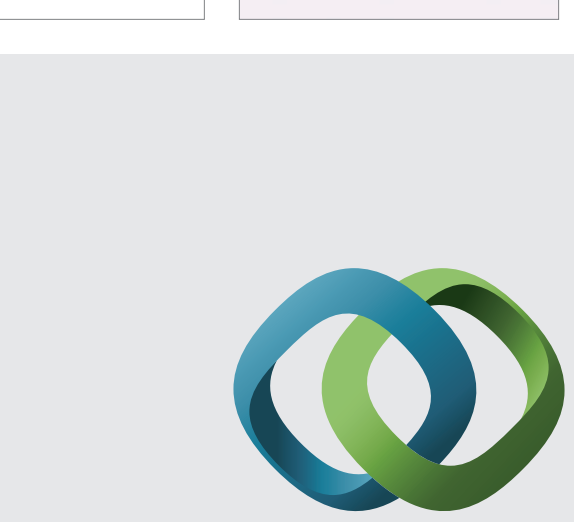

\section{Hindawi}

Submit your manuscripts at

http://www.hindawi.com
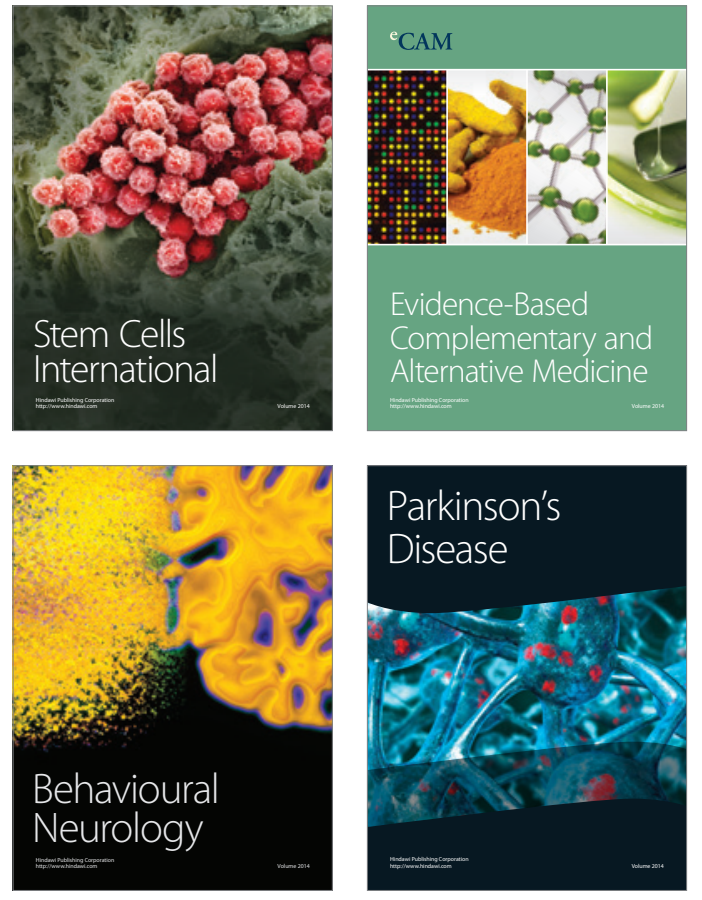
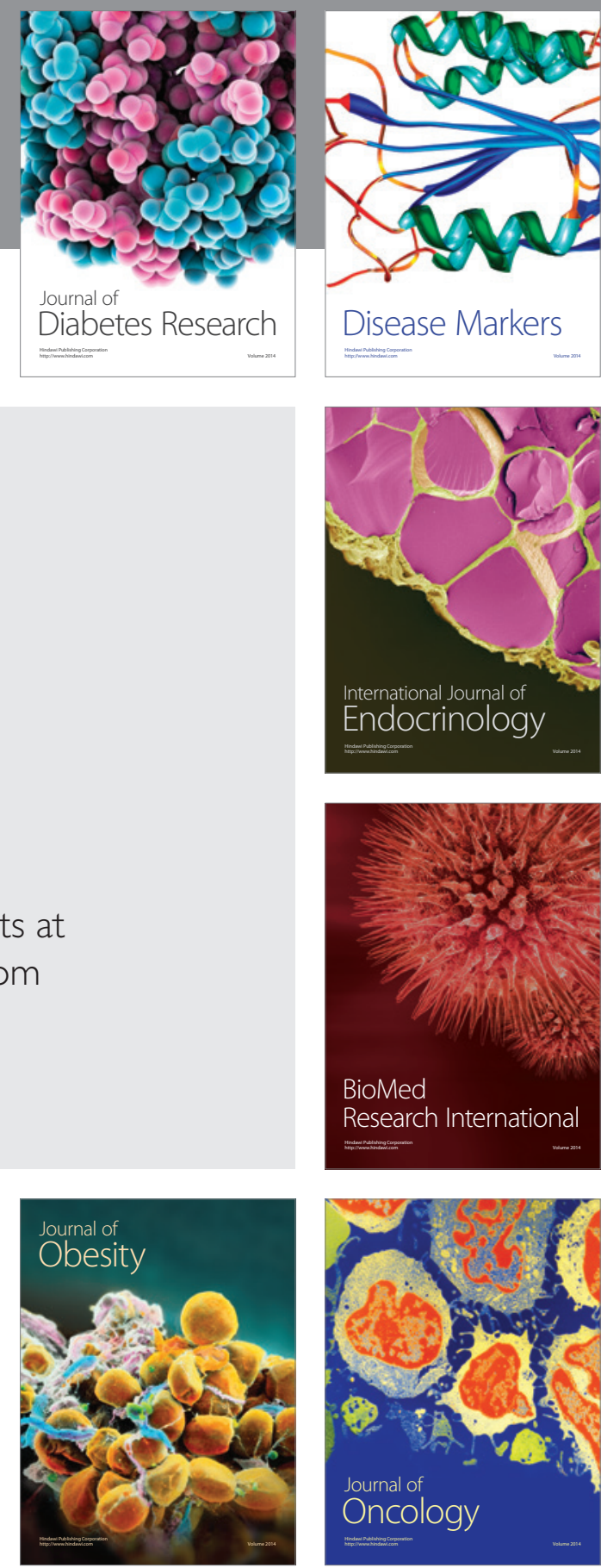

Disease Markers
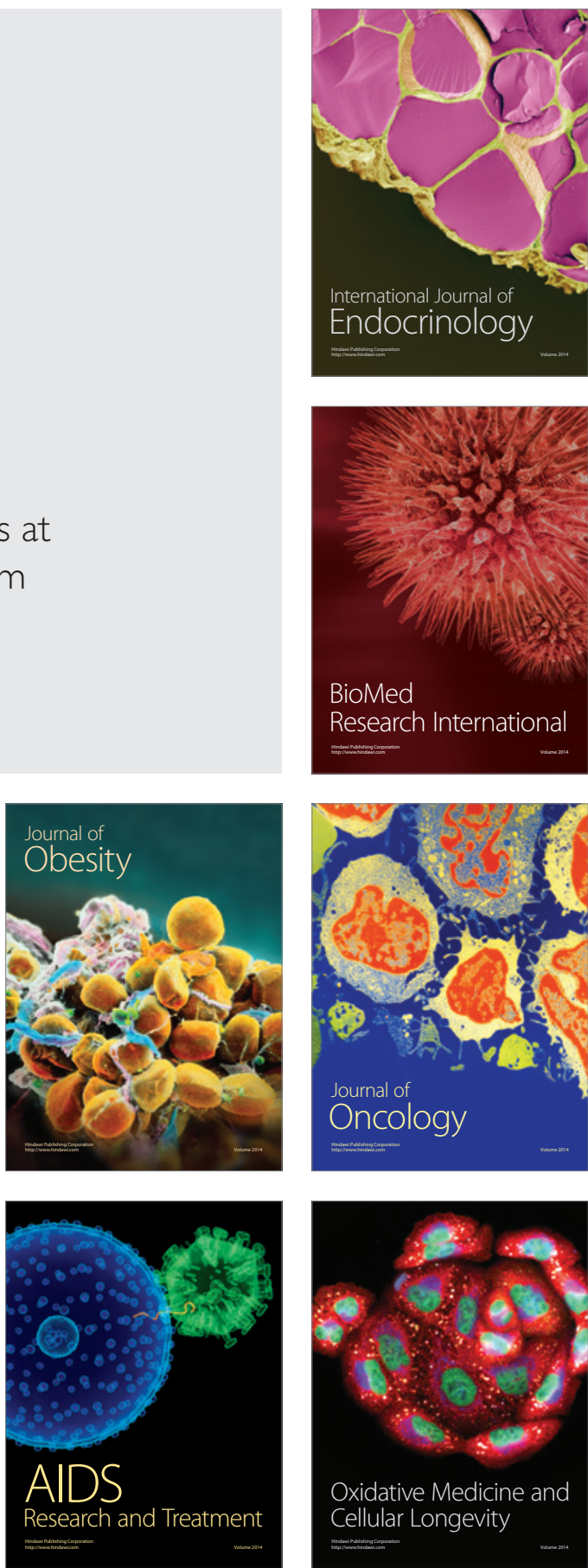